\title{
SIMULATION MODELING OF INTELLIGENT CONTROL ALGORITHMS FOR CONSTRUCTING AUTONOMOUS POWER SUPPLY SYSTEMS WITH IMPROVED ENERGY EFFICIENCY
}

\author{
Ruslan Gimazov ${ }^{1}$, Stanislav Shidlovskiy, ${ }^{2, *}$ \\ ${ }^{1}$ Tomsk Polytechnic University, School of Energy \& Power Engineering, 634050, Tomsk, Russia \\ ${ }^{2}$ Tomsk State University, Faculty of innovative technologies, 634050, Tomsk, Russia
}

\begin{abstract}
The paper considers the issue of supplying autonomous robots by solar batteries. Low efficiency of modern solar batteries is a critical issue for the whole industry of renewable energy. The urgency of solving the problem of improved energy efficiency of solar batteries for supplying the robotic system is linked with the task of maximizing autonomous operation time. Several methods to improve the energy efficiency of solar batteries exist. The use of MPPT charge controller is one these methods. MPPT technology allows increasing the power generated by the solar battery by $15-30 \%$. The most common MPPT algorithm is the perturbation and observation algorithm. This algorithm has several disadvantages, such as power fluctuation and the fixed time of the maximum power point tracking. These problems can be solved by using a sufficiently accurate predictive and adaptive algorithm. In order to improve the efficiency of solar batteries, autonomous power supply system was developed, which included an intelligent MPPT charge controller with the fuzzy logic-based perturbation and observation algorithm. To study the implementation of the fuzzy logic apparatus in the MPPT algorithm, in Matlab/Simulink environment, we developed a simulation model of the system, including solar battery, MPPT controller, accumulator and load. Results of the simulation modeling established that the use of MPPT technology had increased energy production by $23 \%$; introduction of the fuzzy logic algorithm to MPPT controller had greatly increased the speed of the maximum power point tracking and neutralized the voltage fluctuations, which in turn reduced the power underproduction by $2 \%$.
\end{abstract}

\section{Introduction}

One of the main problems for autonomous robotics industry is power supply of robots. One of the possible solution is supplying robots with solar batteries. This method has a number of disadvantages connected with low efficiency of modern solar batteries. However, there are methods to improve the energy efficiency of solar batteries. The use of MPPT charge controller is among these methods.

\footnotetext{
*Corresponding author: shidlovskiy@tpu.ru
} 
Maximum Power Point Tracking (MPPT) is one of the ways to improve the energy efficiency of photovoltaic modules and wind-energetic turbines by obtaining the maximally possible power on output of these systems. MPPT is realized by use of digital devices that analyze the current vs. voltage curve $(\mathrm{CvV})$ in order to determine the optimal voltagecurrent pair, providing the maximum output power.

The most common MPPT algorithm is the perturbation and observation algorithm. In this method, a MPPT device changes the input resistance by a small amount, thus changing the voltage of the solar installation, and measures the power; if power increases - the controller continues to change the voltage in the same direction until the power stops to increase. Figure 1 shows the principle of perturbation and observation algorithm.

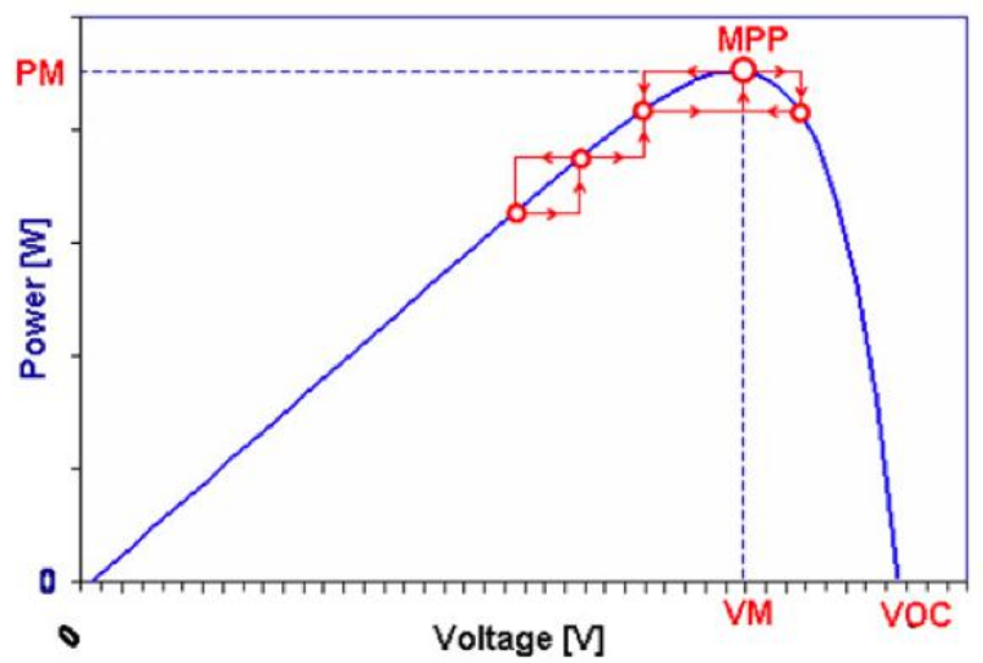

Fig. 1. The principle of the maximum power point tracking according to the perturbation and observation algorithm.

The disadvantages of the perturbation and observation algorithm are the power fluctuations and fixed time of "climbing" (the maximum power point tracking) [1]. Decrease of the "climbing" time leads to the increase in fluctuation amplitude, which leads to the power underproduction. Decrease of the power of fluctuation amplitude leads to the increase of time of the maximum power point tracking. These problems can be solved by using a sufficiently accurate predictive and adaptive algorithm [2, 3].

The urgency of solving the problem of improved energy efficiency of solar batteries for supplying the robotic system is linked with the task of maximizing autonomous operation time.

The aim of this work is to develop an autonomous solar-powered supply system with improved energy efficiency.

The development tasks include system design and simulation.

\section{Development of the autonomous power supply system}

An autonomous robot with the solar-based power system should consist of lightweight and high-strength materials and electronic circuitry that consume little energy. Lower level of 
energy consumption and lower weight of the structure make the use of solar batteries more promising.

There are several options for building the solar-based power systems of robots [4]. The paper considers the development of the combined power system based on solar batteries.

As we have noted above, use of MPPT charge controller can improve energy efficiency of the solar-based power supply system. It is possible to realize such device for an autonomous robot by means of a single-board microcontroller (Arduino, Mbed, etc.). The idea of constructing the charger based on single-board microcontroller for the photovoltaic plant is as follows: Buck-converter, which is controlled by a microcontroller, is used as a charger. When the voltage/current of the photovoltaic module increases, the PWM generator increases pulse frequency, which in turn leads to increase of the output current.

\section{Simulation modeling of the system}

An autonomous robot with the solar-based power system should consist of lightweight and high-strength materials and electronic circuitry that consume little energy. Lower level of energy consumption and lower weight of the structure make the use of solar batteries more promising.

We used MATLAB / Simulink environment to create a model of an autonomous power system.

In order to evaluate effectiveness of the MPPT algorithm and the fuzzy logic-based MPPT algorithm, in addition to the controller itself we designed photovoltaic system including a solar battery, charge controller, accumulator and load. Figure 2 shows the developed model.

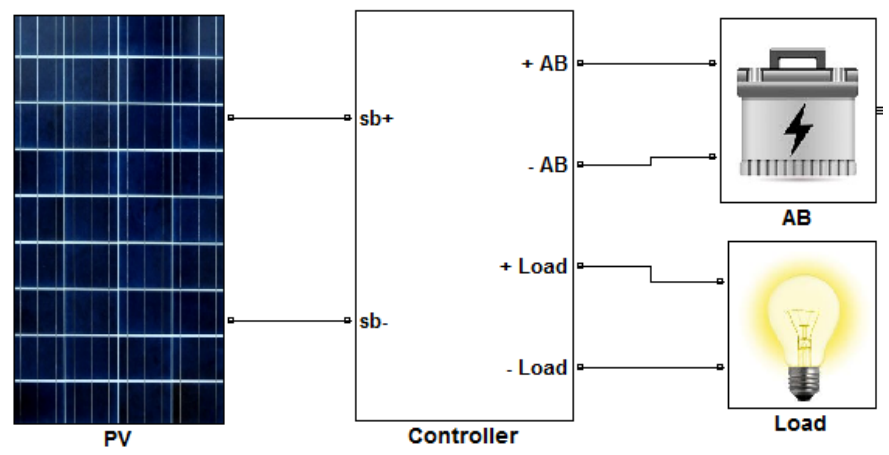

Fig. 2. Model of photovoltaic system.

Perturbation and observation MPPT algorithm is realized in the controller unit. Figure 3 represents this model. 


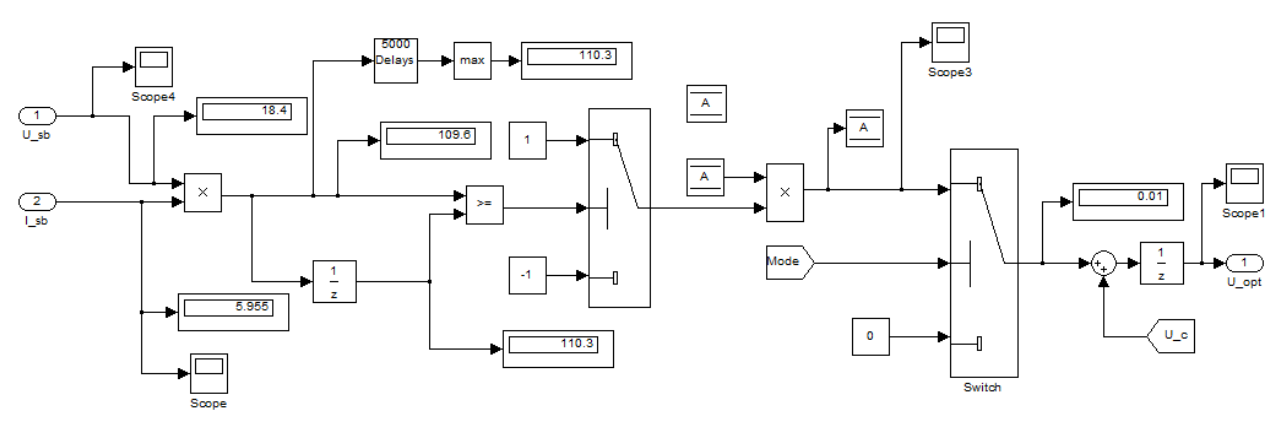

Fig. 3. Model of a common MPPT controller.

Use of the MPPT controller increases the energy production of solar batteries and increases the rate of battery charge. Figure 4 shows comparative graphs of the rate of battery charge (no load).
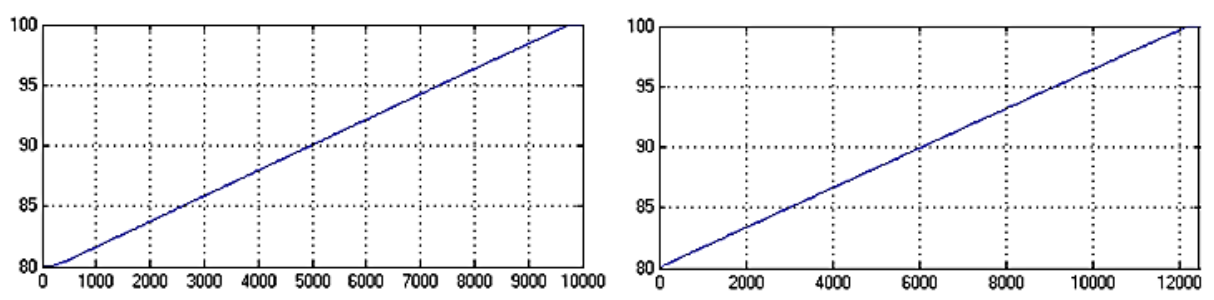

Fig.4. Comparative graphs of the rate of battery charge: left - with use of MPPT; right -with the set voltage.

Figure 4 shows that the rate of battery charge has increased significantly with the MPPT controller. Figure 5 represents the mode of the maximum power point tracking of the developed model.

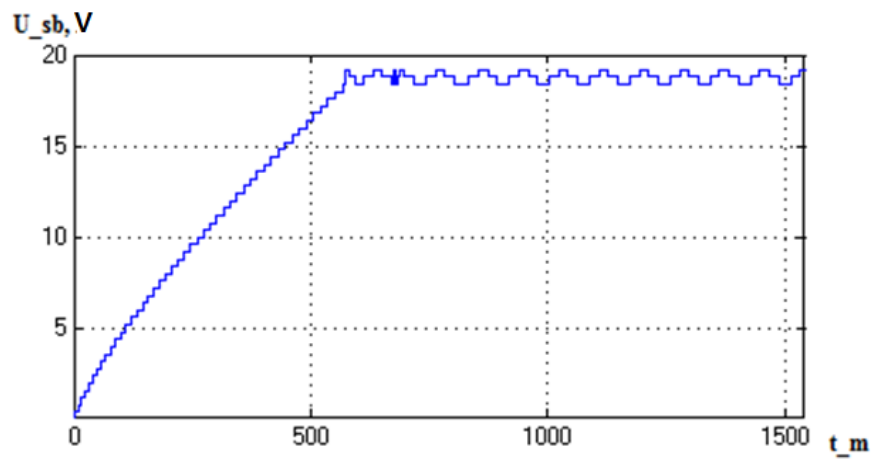

Fig. 5. Mode of maximum power point tracking.

Figure 5 shows that during the algorithm realization, the voltage and thus the power begin to fluctuate; it leads to underutilization of power generated by the solar battery. As we have noted above, this problem can be solved by using the fuzzy logic-based adaptive algorithm. Figure 6 shows a model of the MPPT controller with a fuzzy regulator.

As input linguistic variables of the fuzzy controller, we use the ratio of solar power change to solar voltage change $(\mathrm{E})$, as well as the behavior of $\mathrm{E}(\mathrm{dE})$ : 


$$
\begin{gathered}
E(k)=\left(P_{s b}(k)-P_{s b}(k-1)\right) /\left(V_{s b}\left(k-V_{s b}(k-1)\right)\right. \\
d E(k)=E(k)-E(k-1)
\end{gathered}
$$

The output variable of the fuzzy logic unit is a scaling factor of the step-size MPPT algorithm. Dependence of the output variable value on the input linguistic variables is defined by the set of rules given in table 1 .

Table 1. Rules of the fuzzy controller.

\begin{tabular}{|c|c|c|c|}
\hline $\mathbf{E} \downarrow \backslash \mathbf{d E} \rightarrow$ & low & middle & high \\
\hline low & low & low & low \\
\hline middleL & midL & midL & low \\
\hline middleH & midH & midL & low \\
\hline high & high & midH & midL \\
\hline
\end{tabular}

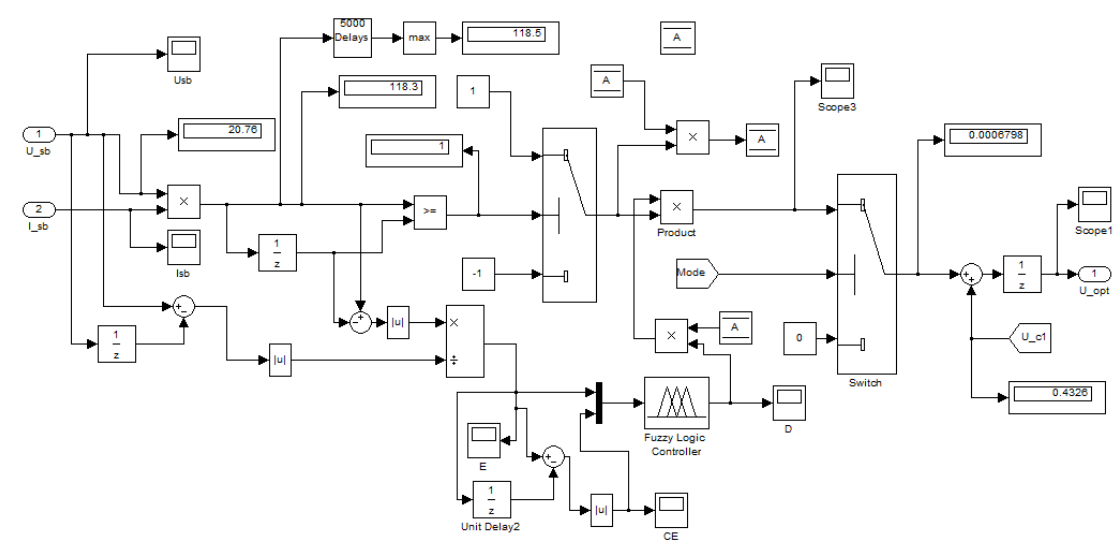

Fig. 6. Model of a fuzzy logic-based MPPT controller.

\section{Study of the influence of the fuzzy logic unit on the MPPT controller}

To study the influence of the fuzzy logic unit on the solar photovoltaic plant, we have set the following initial parameters of the model:

- Normal conditions: temperature $25^{0} \mathrm{C}$, solar insolation level $\mathrm{W}=1000 \mathrm{kWh} / \mathrm{m}^{2}$, the angle of incidence $\alpha=90^{\circ}$.

- Consumer load is turned off.

Figures 7, 8 represent results of modeling.

Figure 7 shows that the use of the fuzzy logic unit in the MPPT controller has greatly increased the speed of the maximum power point tracking and reduced voltage fluctuations, which in turn neutralized the power fluctuations (Figure 8). 


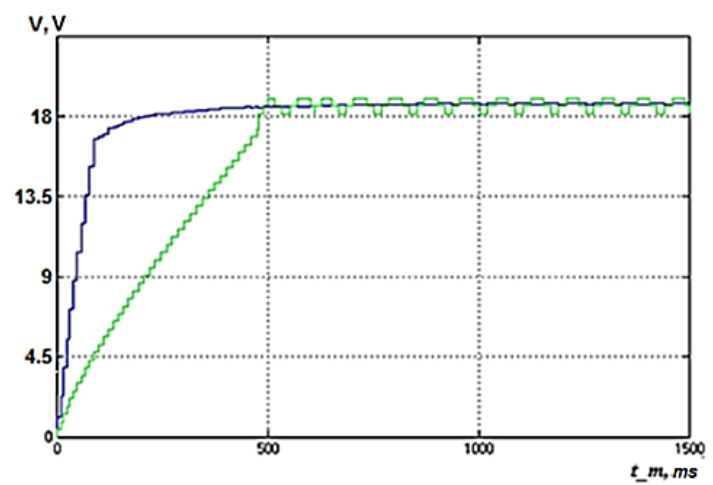

Fig. 7. Voltage comparison of the modes of maximum power point tracking: green - normal MPPT algorithm; blue - fuzzy logic-based algorithm.

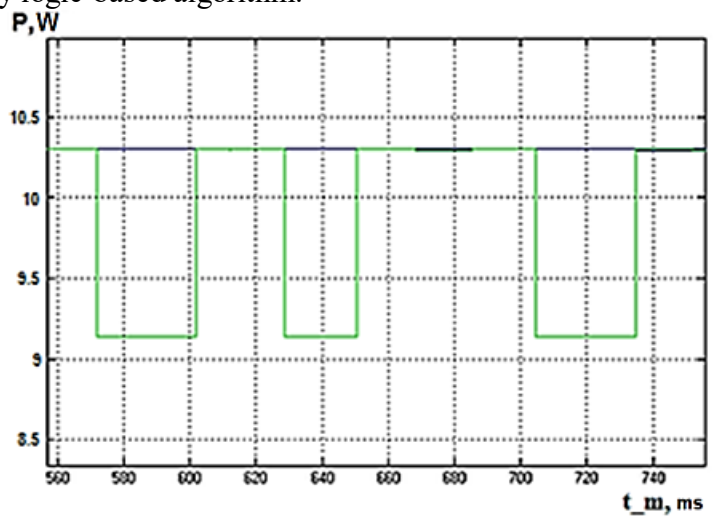

Fig. 8. Power comparison of the modes of maximum power point tracking: green - normal MPPT algorithm; blue - fuzzy logic-based algorithm.

\section{Conclusion}

The described work resulted in development of a solar-based power supply system of autonomous robots. Effectiveness of the developed system is improved by the use of the MPPT charge controller with a fuzzy logic-based adaptive algorithm. Effectiveness of the perturbation and observation MPPT algorithm with adaptation was studied by means of simulation modelling, which proved the model adequacy. The comparative characteristics of the controller with the fuzzy logic and without it were obtained. The developed fuzzy control system increased the speed of the maximum power point tracking and neutralized the power fluctuations.

Simulation modelling established facts that the use of MPPT technology increased energy production by $23 \%$, introduction of the fuzzy logic-based algorithm into the MPPT controller had greatly increased the speed of the maximum power point tracking, neutralized the voltage fluctuations, which in turn reduced the power underproduction by $2 \%$.

\section{Acknowledgment}

The following research has been financially supported by Russian Foundation for Basic Research (RFBR) 16-07-01138 A "Intelligent Reconfigurable Control Systems, Navigation and Image Processing for Autonomous Mobile Robots." 


\section{Reference}

[1] V.A. Russkin, S.M.Semenov, R.K. Dixon, Bulletin of TPU. 4, 78 (2016)

[2] S.V. Shidlovskiy, Automatic control. Reconfigurable structures. (Tomsk State University, Tomsk, 2006)

[3] D.V. Shashev, MATEC Web Conf. 79, (2016)

[4] D. Lovin, How to create android robot by own hands (DMK-Press, Moscow, 2007) 